PROCEEDINGS OF THE AMERICAN MATHEMATICAL SOCIETY

Volume 124, Number 7, July 1996

\title{
GENERALIZED INTERPOLATION IN A MULTIPLY CONNECTED REGION
}

\author{
HARI BERCOVICI AND ADELE ZUCCHI
}

(Communicated by Palle E. T. Jorgensen)

\begin{abstract}
In this paper we extend to the case of multiply connected regions the famous result of Sarason concerning the characterization of operators commuting with the compression of the unilateral shift on $H^{2}$ to a co-invariant subspace.
\end{abstract}

\section{INTRODUCTION}

Let $H^{2}$ denote the usual Hardy space for the unit disk, and let $U_{+}$denote the unilateral shift on $H^{2}$. Assume that $\mathcal{H} \subset H^{2}$ is an invariant subspace for $U_{+}^{*}$, and $T \in \mathcal{L}(\mathcal{H})$ is defined by $T=P_{\mathcal{H}} U_{+} \mid \mathcal{H}$. It was proved by Sarason [10] that every operator $X \in \mathcal{L}(\mathcal{H})$, commuting with $T$, has the form $X=P_{\mathcal{H}} Y \mid \mathcal{H}$, where $Y$ is an analytic Toeplitz operator. Moreover, $Y$ can be chosen so that $\|Y\|=\|X\|$. This fact was then shown to extend several classical interpolation theorems for the unit disk. Our purpose here is to extend Sarason's result to the case when the unit disk is replaced by a multiply connected region in $\mathbf{C}$. The result we obtain is somewhat weaker in the sense that we can only choose $Y$ so that $\|Y\| \leq k\|X\|$, where $k \geq 1$ only depends on the region; on the other hand the results of Ball and Clancey [4] imply that $k>1$ for an annulus. Our proof was inspired by Abrahamse's approach to the Nevanlinna-Pick interpolation problem [1].

Sarason's generalized interpolation theorem is of course a particular case of the Sz.-Nagy-Foias commutant lifting theorem [8]. One may hope that a commutant lifting result can be proved in the multiply connected case. The methods needed for proving such a result must however be quite different from those used for the unit disk, and the theory of bundle shifts [3] would certainly play a role in this development. In the language of bundle shifts, an essential ingredient of our proof here can be stated as follows. All bundle shifts of dimension one are similar, and the similarity can be chosen to be bounded by a constant which only depends on the region. It was of course shown in [3] that bundle shifts of equal dimension are similar, and it would be interesting to know whether the similarity can be chosen to be uniformly bounded, as in the case of dimension one.

Received by the editors November 28, 1994 and, in revised form, January 27, 1995.

1991 Mathematics Subject Classification. Primary 47A45; Secondary 47B35, 30D55, 30E05.

The first author was supported in part by grants from the National Science Foundation.

The second author was supported in part by the Istituto Nazionale di Alta Matematica "F. Severi" of Italy. 


\section{THE MAIN RESULT}

Consider a bounded region $\Omega \subset \mathbf{C}$ whose boundary $\partial \Omega$ consists of $m+1$ disjoint, closed, analytic Jordan curves. These curves will be denoted by $\Gamma_{0}, \Gamma_{1}, \ldots, \Gamma_{m}$, with $\Gamma_{0}$ the outer boundary of $\Omega$.

The theory of Hardy spaces over multiply connected regions has been first studied by Rudin [9]; cf. also [6]. Briefly, a holomorphic function $f$ on $\Omega$ is in $H^{2}(\Omega)$ if the subharmonic function $|f|^{2}$ has a harmonic majorant on $\Omega$. For a fixed $z_{0} \in \Omega$, there is a norm on $H^{2}(\Omega)$ defined by

$$
\|f\|=\inf \left\{u\left(z_{0}\right)^{1 / 2}: u \text { is a harmonic majorant of }|f|^{2}\right\} .
$$

Let $\omega$ be the harmonic measure on $\partial \Omega$ for the point $z_{0}$. Each function $f \in H^{2}(\Omega)$ has nontangential boundary values $f^{*}$ almost everywhere $\mathrm{d} \omega$, and $f^{*}$ is in $L^{2}(\partial \Omega, \omega)$. The mapping $f \rightarrow f^{*}$ is an isometry from $H^{2}(\Omega)$ onto a closed subspace of $L^{2}(\partial \Omega, \omega)$. We shall employ the same letter $f$ to stand both for the function defined on $\Omega$ and for its boundary values. In this way $H^{2}(\Omega)$ can be viewed as a closed subspace of $L^{2}(\partial \Omega, \omega)$.

A function $f$ defined on $\Omega$ is in $H^{\infty}(\Omega)$ if it is holomorphic and bounded. $H^{\infty}(\Omega)$ is a closed subspace of $L^{\infty}(\partial \Omega, \omega)$ and it is a Banach algebra if endowed with the supremum norm. Finally, the mapping $f \rightarrow f^{*}$ is an isometry of $H^{\infty}(\Omega)$ onto a weak ${ }^{*}$-closed subalgebra of $L^{\infty}(\partial \Omega, \omega)$, hence $H^{\infty}(\Omega)$ can be viewed as a weak*closed subalgebra of $L^{\infty}(\partial \Omega, \omega)$. Given two functions $f$ and $g$ in $H^{\infty}(\Omega)$, we write $f \mid g$ if $f$ divides $g$.

We recall from Royden [7] that a function $\theta \in H^{\infty}(\Omega)$ is said to be inner if $|\theta|$ is essentially constant on each component of $\partial \Omega$. It is known that any weak*-closed ideal of $H^{\infty}(\Omega)$ is principal and is generated by an inner function. Of course two inner functions $\theta_{1}$ and $\theta_{2}$ generate the same ideal if and only if $\theta_{1} / \theta_{2}$ and $\theta_{2} / \theta_{1}$ belong to $H^{\infty}(\Omega)$. If this condition is satisfied we say that $\theta_{1}$ and $\theta_{2}$ are equivalent.

Given an inner function $\theta$, the subspace $\theta H^{2}(\Omega)$ of $H^{2}(\Omega)$ is closed, invariant under multiplication by functions in $H^{\infty}(\Omega)$, and it only depends on the equivalence class of $\theta$. Let us denote by $S \in \mathcal{L}\left(H^{2}(\Omega)\right)$ the operator defined by

$$
(S f)(z)=z f(z), \quad z \in \Omega .
$$

We set $\mathcal{H}(\theta)=H^{2}(\Omega) \ominus \theta H^{2}(\Omega)$, and denote by $S(\theta)$ the compression of $\mathrm{S}$ to $\mathcal{H}(\theta)$, i.e.,

$$
S(\theta) f=P_{\mathcal{H}(\theta)} S f, \quad f \in \mathcal{H}(\theta),
$$

where $P_{\mathcal{H}(\theta)}$ denotes the orthogonal projection onto $\mathcal{H}(\theta)$. The operators $S(\theta)$ play the role of Jordan blocks in the theory of operators of class $C_{0}$ on $\Omega$ (see [11]).

For $\varphi \in L^{\infty}(\partial \Omega, \omega)$, the Toeplitz operator $T_{\varphi}$ with symbol $\varphi$ is defined by $T_{\varphi}(f)=P_{H^{2}(\Omega)}(\varphi f), f \in H^{2}(\Omega)$, where $P_{H^{2}(\Omega)}$ is the orthogonal projection of $L^{2}(\partial \Omega, \omega)$ onto $H^{2}(\Omega)$.

Let $\theta, \theta^{\prime}$ be two inner functions in $H^{\infty}(\Omega)$; it is easy to verify that every $u \in$ $H^{\infty}(\Omega)$ which satisfies $\theta^{\prime} \mid u \theta$ determines an operator $X \in \mathcal{L}\left(\mathcal{H}(\theta), \mathcal{H}\left(\theta^{\prime}\right)\right)$ such that $X S(\theta)=S\left(\theta^{\prime}\right) X$, by the formula

$$
X=P_{\mathcal{H}\left(\theta^{\prime}\right)} T_{u} \mid \mathcal{H}(\theta) .
$$

The main result of this paper is a converse of this fact. 
Theorem. There exists a constant $k \geq 1$, depending only on $\Omega$, with the following property. Given two inner functions $\theta, \theta^{\prime} \in H^{\infty}(\Omega)$, and an operator $X \in$ $\mathcal{L}\left(\mathcal{H}(\theta), \mathcal{H}\left(\theta^{\prime}\right)\right)$ satisfying $X S(\theta)=S\left(\theta^{\prime}\right) X$, there exists $u \in H^{\infty}(\Omega)$ such that:

(i) $\|u\|_{\infty} \leq k\|X\|$;

(ii) $\theta^{\prime} \mid u \theta$; and

(iii) $X=P_{\mathcal{H}\left(\theta^{\prime}\right)} T_{u} \mid \mathcal{H}(\theta)$.

Observe that the existence of a constant $k$ as in (i) can be deduced from the open mapping theorem. Our proof yields an estimate of $k$ in terms of the uniform bounds of a certain family of harmonic functions on $\Omega$. As we pointed out before, the best constant $k$ must be greater than one in case $\Omega$ is an annulus, even when the space $\mathcal{H}(\theta)$ is finite-dimensional [4]. Also note that condition (ii) is automatically satisfied when $\theta^{\prime}=\theta$.

The proof uses a modified form of Lemma 5 in [1]. The argument depends on Proposition 1.15 in [2], and is included here for the reader's convenience. In the following $\mathcal{M}^{\perp}$ denotes the orthogonal complement of a subspace $\mathcal{M}$ in $L^{2}(\partial \Omega, \omega)$.

Lemma. There exists a constant $c \geq 1$, depending only on $\Omega$, with the following property. Given any inner function $\theta \in H^{\infty}(\Omega)$ and any $f \in\left(\theta H^{2}(\Omega)\right)^{\perp} \cap$ $L^{\infty}(\partial \Omega, \omega)$, there exist $g \in H^{2}(\Omega)$ and $h \in\left(\theta H^{2}(\Omega)\right)^{\perp}$ such that

(i) $f=\bar{g} h$; and

(ii) $c^{-1}|f| \leq|g|^{2} \leq c|f|, \quad c^{-1}|f| \leq|h|^{2} \leq c|f|$ a.e. on $\partial \Omega$.

Proof. Let $H$ be the set of real harmonic functions in $\Omega$, which are continuous in $\bar{\Omega}$ and constant on each $\Gamma_{j}$, for $j=0,1, \ldots, m$. Let $\Lambda: H \rightarrow \mathbf{R}^{m}$ be defined by $\Lambda(w)=\left(\int_{\Gamma_{1}} d^{*} w, \ldots, \int_{\Gamma_{m}} d^{*} w\right)$. Thus the $j^{t h}$ coordinate of $\Lambda(w)$ is the period about $\Gamma_{j}$ of the conjugate differential $d^{*} w=(\partial w / \partial x) d y-(\partial w / \partial y) d x$ of $w$. It is well-known (see [2]) that $\Lambda$ is surjective, and therefore there exists a positive constant $\gamma$ with the following property: for every $\beta \in \mathbf{R}^{m}$ there exists $w \in H$ such that $\Lambda(w)=\beta$ and $\|w\|_{\infty} \leq \gamma\|\beta\|$.

Suppose now that $f$ is bounded and orthogonal to $\theta H^{2}(\Omega)$. Then $\bar{\theta} f$ is orthogonal to $H^{2}(\Omega)$ and hence $\log |\bar{\theta} f|$ is in $L^{1}(\partial \Omega, \omega)$. It follows that $1 / 2 \log |f|$ is in $L^{1}(\partial \Omega, \omega)$ and thus there exists a harmonic function $u$ in $\Omega$ with boundary values $1 / 2 \log |f|$ a.e. (take $u=\int_{\partial \Omega} 1 / 2 \log |f(\zeta)| d \omega_{z}(\zeta)$ ). Let $\beta \in \mathbf{R}^{m}$ be such that its $j^{\text {th }}$ coordinate $\beta_{j}$ is the period about $\Gamma_{j}$ of the conjugate differential of $u$. We can find a vector $\beta^{0} \in R^{m}$ such that $\left(\beta^{0}-\beta\right)_{j} / 2 \pi$ is an integer and $\beta_{j}^{0} \in[-\pi, \pi)$ for $j=1, \ldots, m$. By the first part of the proof, there exists $w \in H$ such that

$$
\Lambda(w)=\beta^{0} \quad \text { and } \quad\|w\|_{\infty} \leq \pi m^{1 / 2} \gamma .
$$

Set $v=u-w$ and $g=\exp \left(v+i^{*} v\right)$, where ${ }^{*} v$ is the harmonic conjugate of $v$. Then $g$ is single-valued on $\Omega$, it is an outer function in $H^{2}(\Omega)$, and

$$
|g|^{2}=\exp (2 v)=|f| \exp (-2 w)
$$

a.e. on $\partial \Omega$. Set $h=f / \bar{g}$. Since $|h|^{2}=|f|^{2} /|g|^{2}=|f| \exp (2 w)$, and the estimate on $w$ does not depend on $f$ or $\theta$, we have proved (i) and (ii). It remains to show that $h$ is orthogonal to $\theta H^{2}(\Omega)$. Since $g$ is outer, $g H^{\infty}(\Omega)$ is a dense subspace of $H^{2}(\Omega)$ (cf. [6], Proposition 5.4.6). Thus, it suffices to show that $h$ is orthogonal to $\theta g H^{\infty}(\Omega)$. Indeed, if $r \in H^{\infty}(\Omega)$, then $(h, r \theta g)=(h \bar{g}, r \theta)=(f, r \theta)=0$, because $f$ is in $\left(\theta H^{2}(\Omega)\right)^{\perp}$. 
Proof of the Theorem. For every $g \in H^{\infty}(\Omega)$ we can define an operator $g(S(\theta)) \in$ $\mathcal{L}(\mathcal{H}(\theta))$ by the formula $g(S(\theta))=P_{\mathcal{H}(\theta)} T_{g} \mid \mathcal{H}(\theta)$. It is easy to see that $g(S(\theta))$ is a limit in the weak operator topology of rational functions of $S(\theta)$. Now fix $X \in$ $\mathcal{L}\left(\mathcal{H}(\theta), \mathcal{H}\left(\theta^{\prime}\right)\right)$ such that $X S(\theta)=S\left(\theta^{\prime}\right) X$, and note that we also have $X g(S(\theta))=$ $g\left(S\left(\theta^{\prime}\right)\right) X$ for every $g \in H^{\infty}(\Omega)$.

For every $f \in\left(\theta^{\prime} H^{2}(\Omega)\right)^{\perp} \cap L^{\infty}(\partial \Omega, \omega)$ set

$$
\varphi(f)=\left(f, X P_{\mathcal{H}(\theta)} \mathbf{1}\right),
$$

where 1 denotes the constant function in $H^{2}(\Omega)$ with values equal to 1 . If $f$ is factored as in the preceding lemma (with $\theta^{\prime}$ in place of $\theta$ ), then

$$
\begin{gathered}
\varphi(f)=\left(f, X P_{\mathcal{H}(\theta)} \mathbf{1}\right)=\left(\bar{g} h, X P_{\mathcal{H}(\theta)} \mathbf{1}\right) \\
=\left(h, g X P_{\mathcal{H}(\theta)} \mathbf{1}\right)=\left(P_{H^{2}(\Omega)} h, g X P_{\mathcal{H}(\theta)} \mathbf{1}\right) \\
=\left(P_{\mathcal{H}\left(\theta^{\prime}\right)} h, g X P_{\mathcal{H}(\theta)} \mathbf{1}\right)=\left(P_{\mathcal{H}\left(\theta^{\prime}\right)} h, P_{\mathcal{H}\left(\theta^{\prime}\right)} g X P_{\mathcal{H}(\theta)} \mathbf{1}\right) \\
=\left(P_{\mathcal{H}\left(\theta^{\prime}\right)} h, g\left(S\left(\theta^{\prime}\right)\right) X P_{\mathcal{H}(\theta)} \mathbf{1}\right)=\left(P_{\mathcal{H}\left(\theta^{\prime}\right)} h, X g(S(\theta)) P_{\mathcal{H}(\theta)} \mathbf{1}\right),
\end{gathered}
$$

where we used in the fifth equality the fact that $h$ is orthogonal to $\theta^{\prime} H^{2}(\Omega)$. Observe next that

$$
g(S(\theta)) P_{\mathcal{H}(\theta)} \mathbf{1}=P_{\mathcal{H}(\theta)} g P_{\mathcal{H}(\theta)} \mathbf{1}=P_{\mathcal{H}(\theta)} g,
$$

because $g\left(\mathbf{1}-P_{\mathcal{H}(\theta)} \mathbf{1}\right)$ belongs to $\mathcal{H}(\theta)^{\perp}$. Thus

$$
\varphi(f)=\left(P_{\mathcal{H}\left(\theta^{\prime}\right)} h, X P_{\mathcal{H}(\theta)} g\right),
$$

and therefore

$$
|\varphi(f)| \leq\|X\|\|h\|_{2}\|g\|_{2} \leq c^{2}\|X\|\|f\|_{1} .
$$

By the Hahn-Banach theorem, $\varphi$ can be extended to a functional of norm less than or equal to $c^{2}\|X\|$ on $L^{1}(\partial \Omega, \omega)$, and therefore there is $u \in L^{\infty}(\partial \Omega, \omega)$ such that $\|u\| \leq c^{2}\|X\|$ and

$$
\varphi(f)=\left(f, X P_{\mathcal{H}(\theta)} \mathbf{1}\right)=(f, u)
$$

for every $f \in\left(\theta^{\prime} H^{2}(\Omega)\right)^{\perp} \cap L^{\infty}(\partial \Omega, \omega)$. Since $\left(\theta^{\prime} H^{2}(\Omega)\right)^{\perp} \cap L^{\infty}(\partial \Omega, \omega)$ is dense in $\left(\theta^{\prime} H^{2}(\Omega)\right)^{\perp}$ (by a modified version of Theorem 1.7 in [2]), $X P_{\mathcal{H}(\theta)} \mathbf{1}-u$ is orthogonal in $L^{2}(\partial \Omega, \omega)$ to $\left(\theta^{\prime} H^{2}(\Omega)\right)^{\perp}$. Therefore $X P_{\mathcal{H}(\theta)} \mathbf{1}-u=\theta^{\prime} v$ for some $v \in H^{2}(\Omega)$, and hence $u=X P_{\mathcal{H}(\theta)} \mathbf{1}-\theta^{\prime} v \in H^{2}(\Omega)$. We conclude that $u \in H^{\infty}(\Omega)$. Since $\|u\| \leq k\|X\|$ with $k=c^{2}$, it remains to show that $X=P_{\mathcal{H}\left(\theta^{\prime}\right)} T_{u} \mid \mathcal{H}(\theta)$ and $\theta^{\prime} \mid \theta u$. Indeed, if $g \in H^{2}(\Omega) \cap L^{\infty}(\partial \Omega, \omega)$ and $f \in \mathcal{H}\left(\theta^{\prime}\right)$, we have

$$
\begin{gathered}
(f, \theta g u)=\left(f, \theta g X P_{\mathcal{H}(\theta)} \mathbf{1}\right)-\left(f, \theta g \theta^{\prime} v\right) \\
=\left(f, \theta g X P_{\mathcal{H}(\theta)} \mathbf{1}\right)=\left(f, P_{\mathcal{H}\left(\theta^{\prime}\right)}\left(\theta g X P_{\mathcal{H}(\theta)} \mathbf{1}\right)\right) \\
=\left(f,(\theta g)\left(S\left(\theta^{\prime}\right)\right) X P_{\mathcal{H}(\theta)} \mathbf{1}\right)=\left(f, X(\theta g)(S(\theta)) P_{\mathcal{H}(\theta)} \mathbf{1}\right)=0,
\end{gathered}
$$

because $\theta(S(\theta))=0$. We conclude that $\theta u H^{\infty}(\Omega) \subset \theta^{\prime} H^{2}(\Omega)$ and hence $\theta^{\prime} \mid \theta u$.

Finally, for $f \in \mathcal{H}\left(\theta^{\prime}\right)$ and $g \in H^{\infty}(\Omega)$ we have

$$
\begin{gathered}
(f, u g)=\left(f, g X P_{\mathcal{H}(\theta)} \mathbf{1}\right)-\left(f, g \theta^{\prime} v\right) \\
=\left(f, g X P_{\mathcal{H}(\theta)} \mathbf{1}\right)=\left(f, P_{\mathcal{H}\left(\theta^{\prime}\right)}\left(g X P_{\mathcal{H}(\theta)} \mathbf{1}\right)\right)
\end{gathered}
$$




$$
=\left(f, g\left(S\left(\theta^{\prime}\right)\right) X P_{\mathcal{H}(\theta)} \mathbf{1}\right)=\left(f, X g(S(\theta)) P_{\mathcal{H}(\theta)} \mathbf{1}\right)=\left(f, X P_{\mathcal{H}(\theta)} g\right) .
$$

We conclude that $X P_{\mathcal{H}(\theta)} g=P_{\mathcal{H}\left(\theta^{\prime}\right)}(u g)$ and since $\theta^{\prime} \mid u \theta$,

$$
P_{\mathcal{H}\left(\theta^{\prime}\right)}(u g)-P_{\mathcal{H}\left(\theta^{\prime}\right)}\left(u P_{\mathcal{H}(\theta)} g\right)=P_{\mathcal{H}\left(\theta^{\prime}\right)}\left(u\left(g-P_{\mathcal{H}(\theta)} g\right)\right)=0 .
$$

Thus $X h=P_{\mathcal{H}\left(\theta^{\prime}\right)} u h$ whenever $h=P_{\mathcal{H}(\theta)} g$ for some $g \in H^{\infty}(\Omega)$. Since $H^{\infty}(\Omega)$ is dense in $H^{2}(\Omega)$, we have $X=P_{\mathcal{H}\left(\theta^{\prime}\right)} T_{u} \mid \mathcal{H}(\theta)$, as claimed.

\section{REFERENCES}

1. M. B. Abrahamse, The Pick Interpolation Theorem for finitely connected domains, Michigan Math. J. 26 (1979), 195-203. MR 80j:30052

2. M. B. Abrahamse, Toeplitz Operators in multiply connected regions, Amer. J. Math. 96 (1974), 261-297. MR 50:14333

3. M. B. Abrahamse and R. G. Douglas, A Class of Subnormal Operators related to multiply connected domains, Advances in Math. 19 (1976), 106-148. MR 53:1327

4. J. A. Ball and K. F. Clancey, Reproducing Kernels for Hardy Spaces on multiply connected domains, Preprint.

5. H. Bercovici, Operator Theory and Arithmetic in $H^{\infty}$, Amer. Math. Soc., Providence, Rhode Island (1988). MR 90e:47001

6. S. Fisher, Function Theory on Planar Domains, a second course in Complex Analysis, Wiley, New York (1983). MR 85d:30001

7. H. L. Royden, Invariant subspaces of $H^{p}$ for multiply connected regions, Pacific J. Math. 134 (1988), 151-172. MR 90a:46056

8. B. Sz.-Nagy and C. Foias, Dilatations des commutants d'opérateurs, C. R. Acad. Sci. Paris Ser. A 266 (1968), 493-495. MR 38:5049

9. W. Rudin, Analytic functions of class $H_{p}$, Trans. Amer. Math. Soc. 78 (1955), 46-66. MR 16: $810 \mathrm{~b}$

10. D. Sarason, Generalized Interpolation in $H^{\infty}$, Trans. Amer. Math. Soc. 127 (1967), 179-203. MR 34:8193

11. A. Zucchi, Ph.D. Dissertation, Indiana University, Bloomington (1994).

Department of Mathematics, Indiana University, Rawles Hall, Bloomington, Indiana 47405-5701

E-mail address: bercovic@indiana.edu 